Research Article

\title{
Determination and Pharmacokinetics of Omeprazole Enantiomers in Human Plasma and Oral Fluid Utilizing Microextraction by Packed Sorbent and Liquid Chromatography-Tandem Mass Spectrometry
}

\author{
Hytham Ahmed, ${ }^{1}$ Abdel-Aziz Wahbi, ${ }^{2}$ Hatem Elmongy, ${ }^{2}$ Ahmad Amini, ${ }^{3}$ Hirsh Koyi, \\ Eva Branden, ${ }^{4}$ and Mohamed Abdel-Rehim ${ }^{5}{ }^{5}$ \\ ${ }^{1}$ Department of Pharmaceutical Analysis, Menoufia University, Menofia Governorate, Egypt \\ ${ }^{2}$ Department of Pharmaceutical Analytical Chemistry, University of Alexandria, Alexandria 21521, Egypt \\ ${ }^{3}$ Swedish Drug Agency, Uppsala, Sweden \\ ${ }^{4}$ Department of Respiratory Medicine Gävle Hospital and Centre for Research at Uppsala University/County \\ Council of Gävleborg, Gävle, Sweden \\ ${ }^{5}$ Karolinska Institute, Solna SE17176, Sweden \\ Correspondence should be addressed to Mohamed Abdel-Rehim; mohamed.astra@gmail.com
}

Received 30 August 2020; Revised 5 December 2020; Accepted 7 January 2021; Published 19 January 2021

Academic Editor: Eladia M. Pena Mendez

Copyright (c) 2021 Hytham Ahmed et al. This is an open access article distributed under the Creative Commons Attribution License, which permits unrestricted use, distribution, and reproduction in any medium, provided the original work is properly cited.

\begin{abstract}
In the present work, the determination of omeprazole (OME) enantiomers in oral fluid and plasma samples was carried out utilizing microextraction by packed sorbent (MEPS) and liquid chromatography-tandem mass spectrometry. A chiral column with cellulose-SB phase was used for the first time for enantiomeric separation of OME with an isocratic elution system using $0.2 \%$ ammonium hydroxide in hexane-ethanol mixture $(70: 30, \mathrm{v} / \mathrm{v})$ as the mobile phase. OME enantiomers were determined utilizing a triple quadrupole tandem mass spectrometer in positive ion mode (ESI+) monitoring mass transitions: $\mathrm{m} / z 346.3 \longrightarrow 198.0$ for OME and $\mathrm{m} / \mathrm{z} 369.98 \longrightarrow 252.0$ for internal standard. The limits of detection and quantification of the present method for both enantiomers were 0.1 and $0.4 \mathrm{ng} / \mathrm{mL}$, respectively. The method validation provided good accuracy and precision. The matrix effect factor was less than $5 \%$, and no interfering peaks were observed. The interday precision values ranged from 2.2 to 7.5 (\%RSD), and the accuracy of determinations varied from $-9.9 \%$ to $8.3 \%$. In addition, the pharmacokinetics (PK) of omeprazole enantiomers in healthy subjects after a single oral dose was investigated. (S)-Enantiomers showed higher levels than (R)-enantiomers throughout $24 \mathrm{~h}$. It was found that the mean maximum concentrations of (R)- and (S)-omeprazole in plasma samples were about two times higher than in oral fluid.
\end{abstract}

\section{Introduction}

Omeprazole (OME) is the first proton pump inhibitor (PPI) that acts by blocking the $\mathrm{H}+-\mathrm{K}+\mathrm{ATPase}$ enzymes (proton pumps) irreversibly. OME is indicated in treatment of all acid-related diseases, where the target of treatment is to reduce gastric acid secretion, such as dyspepsia, peptic ulcer, gastric ulcer, and gastroesophageal reflux disease [1]. It is a chiral drug but used as a racemic mixture and pure enantiomer for medicinal purposes [2]. Commonly, the pharmacodynamics and pharmacokinetics (PK) of a chiral drug are potentially different between the optical isomers. Therefore, it is important to study the individual enantiomers to get clear knowledge about pharmacokinetics and pharmacodynamics of any chiral drug. In OME drug, the (S)-enantiomer (esomeprazole) showed better effect, and it was formulated as an enantio-pure drug by AstraZeneca under the commercial name of Nexium ${ }^{\circledR}$ [3-5]. Solid-phase 
extraction (SPE) is the mainly employed sample preparation technique for extracting OME from plasma [6-9]. In addition, liquid-liquid extraction (LLE) [10] and 96-well LLE [11] have been applied for OME measurement in biological samples. However, SPE provides some advantages, such as selectivity and efficiency, over other techniques, especially LLE [12, 13]. Moreover, achiral-chiral column-switching technique has been used for online precolumn extraction of OME [14]. However, there is an increasing demand for miniaturized and automated sample preparation techniques which are simple, cheap, and provide acceptable drug recovery and selectivity [15].

Microextraction by packed sorbent (MEPS) is a miniaturized SPE technique, in which the volumes of sample and solvents is highly reduced. MEPS is efficient for the removal of contaminants from samples. In MEPS, the packed sorbent is integrated directly into the syringe via sampling needle which could be used several times for biological samples instead of single-use SPE cartridges [16], can be fully automated, and be used for small-volume samples [16-21].

As oral fluid is easier to handle and is less complex than the plasma matrix, it has been a promising matrix for drug analysis and pharmacokinetic studies [22-27]. Oral fluid samples can be easily collected from infants, disabled, or anxious patients [28]. In comparison with plasma, oral fluid can provide shorter sampling time, more simple and noninvasive sampling strategy. Furthermore, daily secretion of human oral fluid is too high (up to $1.5 \mathrm{~L}$ ) which enables sample collection at short intervals $[29,30]$. Therefore, oral fluid can be considered as an ideal biological fluid for drug monitoring.

OME enantiomers have been separated by using chiral columns with polysaccharide-based stationary phases formed of carbamate derivatives of cellulose (Chiralpak $\mathrm{AD}$ column, Chiralcel OJ column, Chiralpak AS column, and Chiralcel OD column) $[7,8,13]$ or amylose $[14,31]$ as well as protein-based chiral stationary phase (bovine serum albu$\min )[6]$.

Several studies have been conducted for investigating the pharmacokinetic parameters of OME and its enantiomers in human plasma after single oral dose [32] or repeated oral doses [33]. Oral fluid samples provide a safe solution for sample collection procedures. The unbound drug molecules, being responsible for the therapeutic activity of the administered drug, are distributed to the oral fluid [34].

The present work describes an enantioselective chromatographic method with mass spectrometric detection for the separation and quantification of OME enantiomers in plasma and oral fluid samples using MEPS for sample preparation and chiral column cellulose-SB tris(3,5dimethylphenylcarbamate) for the chiral separation. The developed method aims to provide a reliable determination of OME enantiomers in oral fluid as an alternative specimen to plasma to monitor the therapeutic concentrations of the drug. To our knowledge, the present study is the first study for the analysis of OME enantiomers in human oral fluid samples, using a cellulose-SBbased stationary phase.

\section{Materials and Methods}

2.1. Chemicals. OME racemate, (R)-OME, (S)-OME, and (S)-lansoprazole (I.S) were purchased from Sigma-Aldrich (Steinheim, Germany). HPLC-grade hexane, ethanol, formic acid, and ammonium hydroxide were purchased from Merck (Darmstadt, Germany). A Milli-Q Plus water purification system from Millipore Corporation (Bedford, USA) was used for water purification.

\subsection{Preparation of Standards and Quality Control Samples.} Two stock solutions $\left(5 \mu \mathrm{g} \cdot \mathrm{mL}^{-1}\right.$, each) were prepared in ethanol (one to prepare calibration standards and one for the quality control (QC) samples), and the standards and QC samples were prepared in blank pooled human plasma (heparin) and oral fluid samples (from six different subjects). The stock solutions showed stable for at least one month at $4^{\circ} \mathrm{C}$. The concentration of the standard samples in plasma was in the range of $25-600 \mathrm{ng} / \mathrm{mL}$ and in oral fluid was $25-300 \mathrm{ng} / \mathrm{mL}$. QC samples were prepared in plasma and oral fluid in three concentration levels: low (QCL), medium $(\mathrm{QCM})$, and high $(\mathrm{QCH})$ as reported in Table 1.

2.3. Instrumentation. The liquid chromatography system (LC) consists of two pumps, Shimadzu LC-10ADvp, (Kyoto, Japan), an autosampler (CTC-Pal, Analytics AG, Zwingen, Switzerland), and a $50 \mu \mathrm{L}$ sample loop. The used chromatographic column was a CHIRAL ART cellulose-SB $(150 \times 4.6 \mathrm{~mm}, 5 \mu \mathrm{m}$ particle size $)$ and was obtained from YMC Europe GmbH (Dinslaken, Germany). Different mobile phase composition utilizing ethanol and hexane with different additives (formic acid and ammonium hydroxide) was investigated. The best chromatographic conditions consisted of $0.2 \%$ ammonium hydroxide in $n$-hexane/ethanol $(70: 30, \mathrm{v} / \mathrm{v})$. The mobile phase flow rate was at $0.8 \mathrm{~mL} /$ min with split of $1: 1$ before the MS interface. A $50 \mu \mathrm{L}$ sample volume was injected. eVol sampling device with MEPS syringe and C8 sorbent pins were supplied by SGE Analytical Science (Melbourne, Australia).

Electrospray ionization in positive ion mode (ESI+) was performed using a triple quadrupole mass spectrometer detector (Quattro-micro, Waters, Manchester, UK). 150 and $400^{\circ} \mathrm{C}$ were the MS source block and desolvation temperatures, respectively. Nitrogen gas was utilized not only for drying $\left(950 \mathrm{Lh}^{-1}\right)$ but also for nebulization $\left(60 \mathrm{Lh}^{-1}\right)$. Argon gas was utilized as collision gas (collision energy $10 \mathrm{eV}$ for $\mathrm{OME}$ and $20 \mathrm{eV}$ for lansoprazole). OME enantiomers analysis in plasma and oral fluid samples was performed by using LCMS/MS with multiple reaction monitoring (MRM) of the transitions: $\mathrm{m} / \mathrm{z} 346.30>198.05$ for OME and $\mathrm{m} / \mathrm{z}$ $369.98>252.02$ for I. S. utilizing a dwell time of $0.2 \mathrm{sec} /$ transition. Peak-area ratios (OME/I. S.) were used for all calculations. Data analysis was done by using MassLynx software (version 4.1).

2.4. Sample Preparation. The QC samples in plasma and oral fluid were prepared and stored at $-20^{\circ} \mathrm{C}$ until the analysis, 
TABLE 1: Accuracy and precision of QC samples in human plasma and oral fluid.

\begin{tabular}{|c|c|c|c|c|c|c|}
\hline \multirow{2}{*}{\multicolumn{2}{|c|}{ Sample matrix drug }} & \multirow{2}{*}{ QC (ng/mL) } & \multicolumn{2}{|c|}{ Intraday $(n=6)$} & \multicolumn{2}{|c|}{ Interday $(n=18)$} \\
\hline & & & RSD (\%) & Accuracy (\%) & RSD (\%) & Accuracy (\%) \\
\hline \multirow{6}{*}{ Plasma } & & 75 & 5.7 & -8.8 & 4.9 & -5.7 \\
\hline & (R) & 250 & 2.4 & -5.9 & 2.2 & -6.1 \\
\hline & & 500 & 5.1 & -10.3 & 6.6 & -2.4 \\
\hline & & 75 & 2.3 & -3.5 & 4.4 & -4.4 \\
\hline & (S) & 250 & 5.0 & -7.0 & 3.2 & -1.9 \\
\hline & & 500 & 5.8 & -7.1 & 5.6 & -7.0 \\
\hline \multirow{6}{*}{ Oral fluid } & & 75 & 5.3 & -5.5 & 5.3 & -3.5 \\
\hline & (R) & 150 & 4.3 & -3.7 & 4.5 & 8.3 \\
\hline & & 250 & 4.6 & -4.0 & 3.5 & -5.4 \\
\hline & & 75 & 5.6 & 5.0 & 7.5 & 4.8 \\
\hline & (S) & 150 & 4.5 & -4.7 & 5.3 & -9.9 \\
\hline & & 250 & 4.2 & 6.9 & 5.0 & -5.7 \\
\hline
\end{tabular}

while the standard calibrations were prepared freshly for each validation assay. A $100 \mu \mathrm{L}$ of each sample was mixed with a $100 \mu \mathrm{L}$ of the I. S (20 $\mu \mathrm{g} \mathrm{mL}-1$ in methanol), and then the samples were diluted with water $(1: 4)$ and centrifuged for few minutes ( $1-3 \mathrm{~min})$. Thereafter, extraction utilizing a programmed eVol device with MEPS syringe contains C8 sorbent. The sorbent was conditioned using $100 \mu \mathrm{L}$ ethanol followed by $100 \mu \mathrm{L}$ of water. The sample was drawn 6 times for preconcentration of the analytes. Then, the sorbent was washed by $5 \%$ methanol in water $(2 \times 100 \mu \mathrm{L})$ two times to take away the interfering materials in the samples. A $500 \mu \mathrm{L}$ ethanol $(2 \times 250 \mu \mathrm{L})$ was utilized for the analyte and I.S. elution. The samples were then subjected to evaporation until dryness under nitrogen, and the residues were reconstituted with $150 \mu \mathrm{L}$ ethanol. The MEPS sorbent was reused after washing 3-4 times with water and 4-5 with ethanol to overcome carry over.

2.5. Method Validation. The validation of the proposed method was carried out according to FDA guidelines [35] including accuracy, precision, matrix effect, selectivity, linearity, limits of detection and quantitation, recovery, and robustness.

Quality control samples at three different levels: low (QCL), medium (QCM), and high $(\mathrm{QCH})(n=6)$ were used for the determination of precision and accuracy of the method. For intraday precision and accuracy, a single analytical batch was analyzed. On the other hand, three different batches with three triplicates were analyzed for the evaluation of the interday precision and accuracy. The accepted data for the precision with relative standard deviation (RSD) should be within $\pm 15 \%$, and the accuracy with relative error (RE) from the nominal values should not exceed $15 \%$ and $20 \%$ for lower limit of quantitation (LLOQ).

The calibration curve for plasma ranged from $25 \mathrm{ng} / \mathrm{mL}$ to $600 \mathrm{ng} / \mathrm{mL}(25,50,100,150,200,250,300$, and $600 \mathrm{ng} /$ $\mathrm{mL}$ ), while for oral fluid, it was in the range of $25-300 \mathrm{ng} / \mathrm{mL}$ $(25,50,100,150,200,250$, and $300 \mathrm{ng} / \mathrm{mL})$. Each back calculated standard concentration should not be more than two-third of the points to be accepted with $\% \mathrm{RSD} \leq 15 \%$ except lowest concentration $(\leq 20 \%)$.

The stability of OME in plasma and oral fluid was performed using triplicates of the QC solutions. Four types of stability studies were done for QC samples. First type was short-term stability by defrost the samples at room temperature for eight hours before analysis time; the second type was long-term stability via keeping the samples at $-20^{\circ} \mathrm{C}$ for fifteen days before analysis time; the third type was three freeze-thaw stability cycles by leaving the iced samples at $-20^{\circ} \mathrm{C}$ to melt at room temperature. After that, samples were restored at $-20^{\circ} \mathrm{C}$, and this procedure was repeated three times before analysis time, and finally, the last type was postpreparative stability of the processed samples by keeping them for $24 \mathrm{~h}$ at $4^{\circ} \mathrm{C}$ before analysis time. If assay results were within the acceptable limits of accuracy $( \pm 15 \%)$ and precision (15\%), then samples could be considered to be stable.

2.6. PK Analysis. The PK parameters were calculated from both oral fluid and plasma samples using the concentrationtime profile data for each enantiomer. $C_{\max }$ and $T_{\max }$ were calculated directly from the concentration-time plot of the individual enantiomer. The elimination half time $\left(t_{1 / 2}\right)$ and the elimination rate constant $\left(k_{\mathrm{e}}\right)$ were estimated from the terminal slope of the plasma/fluid concentration-time profile based on first-order kinetics.

\section{Result and Discussion}

3.1. Method Development. The extraction of OME enantiomers from plasma and oral fluid samples was achieved using MEPS eVol sampling device. Different factors affecting extraction efficiency such as sorbents (C8, C18, and polystyrene), washing, and eluting solutions (methanol, ethanol, and isopropanol) were investigated. Best results were obtained with C8 as sorbent (Figure 1), water as washing solution, and ethanol as eluting solvent (Figure 2).

The chromatographic separation of OME enantiomers was achieved using chiral-SB column packed with cellulose tris-(3,5-dimethylphenylcarbamate) immobilized on silica gel as the chiral selector. Hexane containing different volumes of ethanol or isopropanol was investigated as a mobile phase. Different mobile phase composition consisting of ethanol and hexane with different additives (formic acid and ammonium hydroxide) was investigated. It is known that the addition of formic acid in the mobile phase results in good 


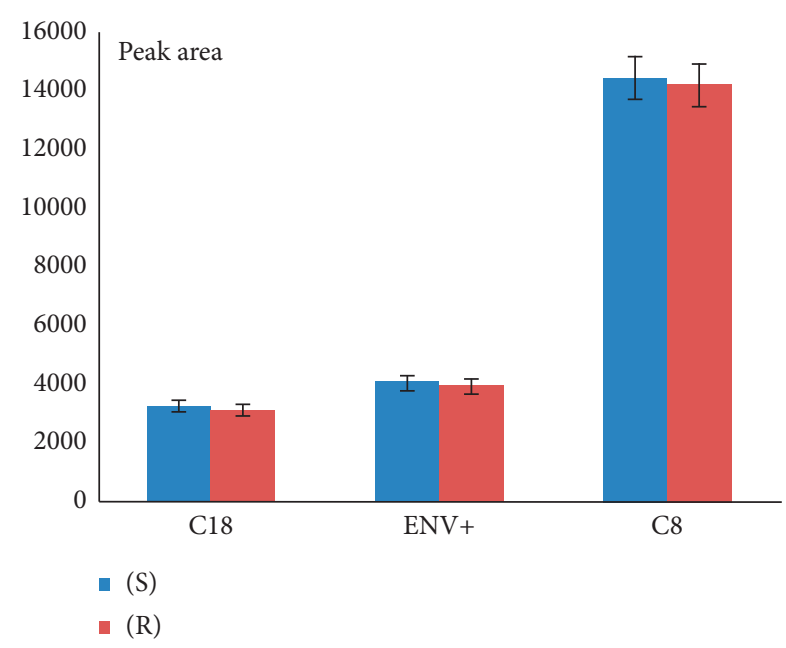

FIGURE 1: Effect of sorbent type on extraction efficiency (sample conc. $0.01 \mu \mathrm{g} \cdot \mathrm{mL}-1)$.

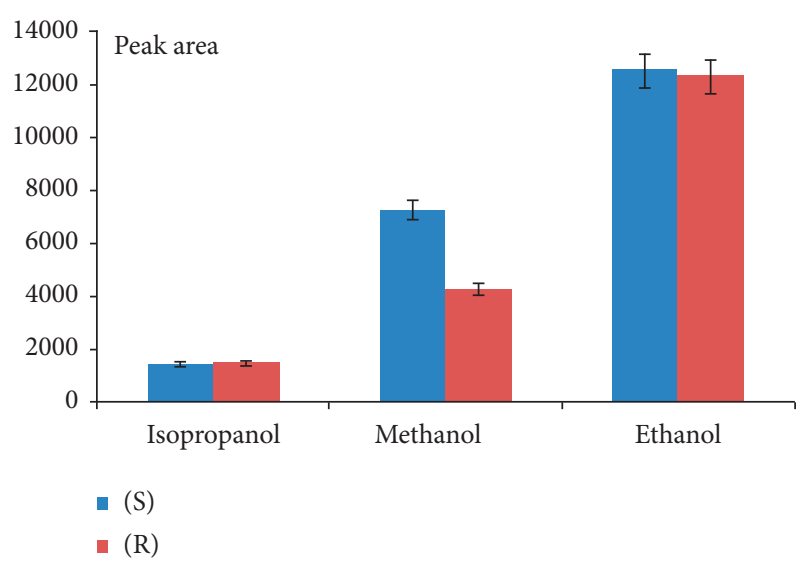

FIGURE 2: Effect of elution solvent on extraction efficiency (C8, sample conc. $0.05 \mu \mathrm{g} \cdot \mathrm{mL}^{-1}$ ).

ionization in ESI interface, but in our case, it did not work well and it resulted in poor peak shape and poor enantioseparation. The addition of ammonium hydroxide on the other hand generated Gaussian peak shape and good MS signal for both the OME enatiomers and internal standard. Thus, the best result was obtained using a mobile phase consisting of n-hexane/ethanol $(70: 30, \mathrm{v} / \mathrm{v})$ and ammonium hydroxide $(0.2 \% \mathrm{v} / \mathrm{v})$. The presence of ammonium hydroxide as a basic modifier in the mobile phase improved the enantiomeric selectivity and peak symmetry (Figure 3 ). The mobile phase flow rate was $0.8 \mathrm{~mL} \cdot \mathrm{min}^{-1}$, and a baseline separation between all analytes was achieved within $10 \mathrm{~min}$ of the run.

The triple quadrupole mass spectrometry was employed for the identification of OME enantiomers. For both enantiomers the precursor and product ions were appeared at 346.30 and $198.05 \mathrm{~m} / z$ values, while the corresponding ions for the internal standard were detected at 369.98 and 252.02 $\mathrm{m} / z$, respectively.
3.2. Method Validation. The validation of the method was run as described previously including linearity, limit of detection (LOD), lower limit of quantification (LLOQ), accuracy, precision, recovery, matrix effects, selectivity, and carry over for determination of OME in human plasma and oral fluid samples in line with FDA guidelines. The calibration curves were prepared in the range of 25-600 $\mathrm{ng} \cdot \mathrm{mL}^{-1}$ and $25-300 \mathrm{ng} \cdot \mathrm{mL}^{-1}$ in human plasma and oral fluid samples, respectively, with LLOQ of $25 \mathrm{ng} / \mathrm{mL}$ and a quadratic equation weighted with $1 / x$ was used for the calculations. The calculated limits of detection $(\mathrm{S} / \mathrm{N} \geq 3)$ and quantitation $(\mathrm{S} / \mathrm{N} \geq 10)$ for both enantiomers in plasma and oral fluid were 0.1 and $0.4 \mathrm{ng} / \mathrm{mL}$, respectively. The accuracy and precision were determined by the analysis of QC samples (QC low, QC medium, and QC high) as mentioned in Table 1.

3.2.1. Calibration. The peak area ratio of each enantiomer to I. S. was plotted against the corresponding concentration for construction of calibration curves for plasma and oral fluid samples. The quality-control samples were treated in the same way as the standards. Quadratic equation was used for the standard curve due to the complexity of the plasma and oral fluid matrices. Using oral fluid and plasma, a close relationship between concentration and peak area ratio (OME/I. S.) was observed in the concentration range $25-600 \mathrm{ng} / \mathrm{mL}$ and $25-300 \mathrm{ng} / \mathrm{mL}$ in plasma and oral fluid, respectively. The correlation coefficient $\left(R^{2}\right)$ values obtained for plasma and oral fluid matrices were $\geq 0.999$.

(S)-Lansoprazole was used as internal standard, and the LLOQ was set to $25 \mathrm{ng} / \mathrm{mL}$. A good correlation between peak area ratios and analyte concentrations was obtained which demonstrated reliability of the method. Figure 4 shows LCMS/MS chromatograms of OME in plasma and oral fluid.

3.2.2. Accuracy and Precision. Table 1 reports the accuracy and precision data from the QC samples in plasma and oral fluid samples. Precision was calculated as the percentage of $\mathrm{RSD}$, and the accuracy was calculated as the percent deviation of the obtained value from nominal value. The method accuracy and precision were within the accepted range, i.e., the accuracy and the precision values for plasma samples ranged from $-1.9 \%$ to $-10.3 \%$ and between 2.2 and $5.8 \%$, respectively, for both intra- and interday assays. For oral fluid samples, the accuracy values varied between $-9.9 \%$ and $8.3 \%$, and the precision ranged from 4.3 to $7.5 \%$ for both intra- and interday assays.

3.2.3. Extraction Recovery. The average absolute recovery for each enantiomer in both plasma and oral fluid samples using QC samples was 95\%.

3.2.4. Matrix Effect and Selectivity. Matrix effects of the tested biological fluids samples of OME enantiomers were shown to be within $95 \%$ and $103 \%$, and thus no significant ion suppression was observed. 


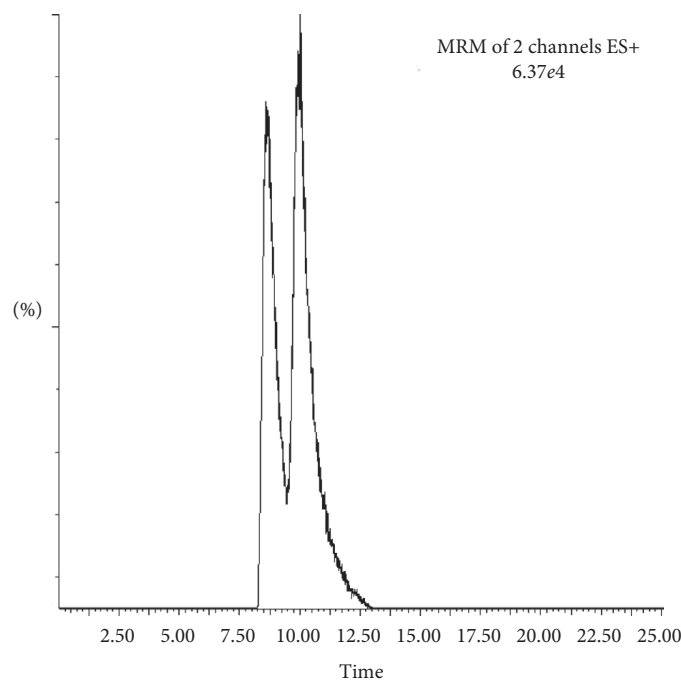

(a)

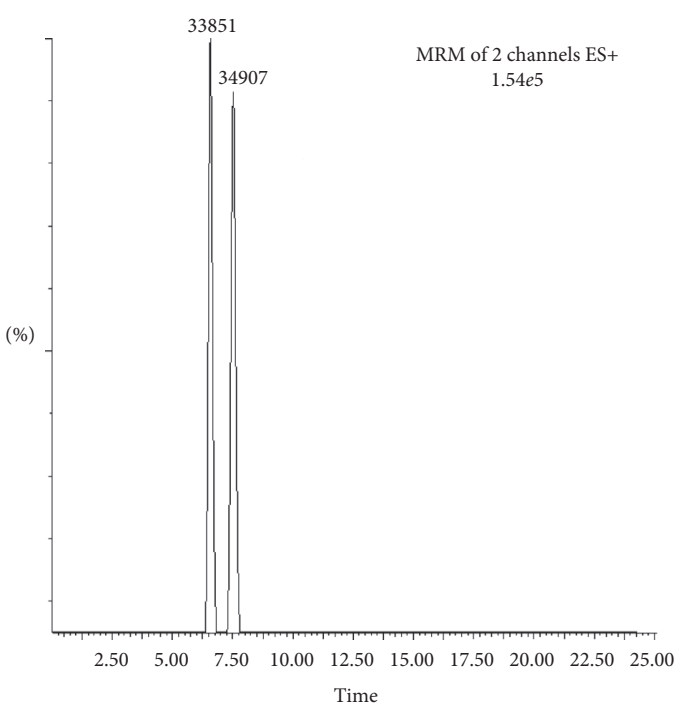

(b)

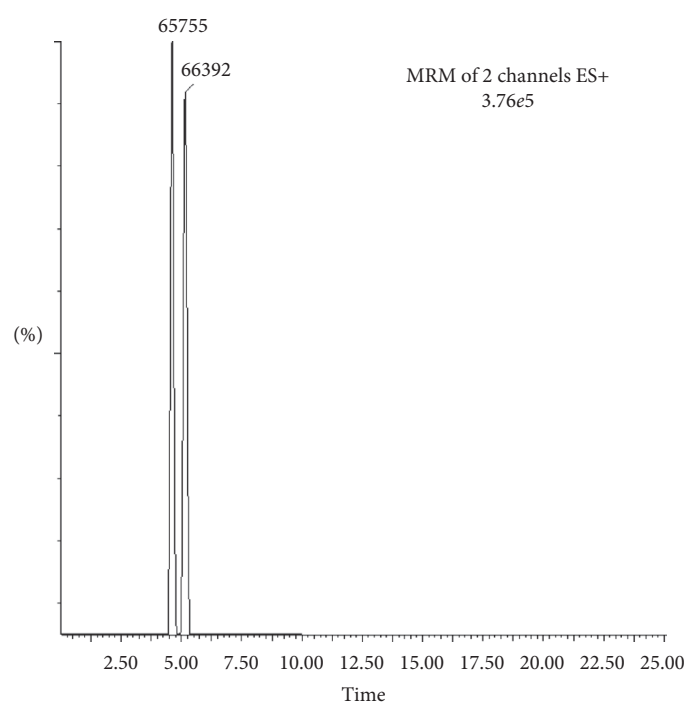

(c)

Figure 3: Effect of mobile phase composition on the separation of OME enantiomers: (a) hexane + isopropanol (80:20) as mobile phase, (b) hexane + ethanol $(80: 20)$, and (c) hexane + ethanol $+\mathrm{NH}_{3} \mathrm{OH}(70: 30: 0.2 \mathrm{v} / \mathrm{v})$.

For the method selectivity, blank plasma and oral fluid samples from different subjects $(n=6)$ were analyzed. The results showed that no interfering peak was detected at the elution positions of at the enantiomer's and I. S.

3.2.5. Stability. The stability studies were conducted analyzing each QC sample in triplicate. No significant change in the enantiomers concentration in short- and long-term being indicated by the mean recovery values was observed. Also, there was no effect on the quantification of target analyte in the three freeze-thaw cycles as well. In addition, the samples were assayed after $24 \mathrm{~h}$ at $4^{\circ} \mathrm{C}$ (postpreparative stability). The obtained data indicated that both used biological samples could be analyzed under such laboratory conditions and after storage at $-20^{\circ} \mathrm{C}$ for up to two weeks without significant change in the drug chemistry or quantity (Table S1).
3.3. Comparison of Developed Method for OME Analysis. Several methods for extraction and analyzing of OME have been developed. In this part, ability of the developed method was compared with other methods, and the results of this part are presented in Table 2.

3.4. Method Application to PK Study of OME in Healthy Individuals. The PK study of OME enantiomers was performed in a group of 4 healthy subjects. The patient's samples were provided by the center of clinical research, Gavle hospital, Sweden. The subjects were 3 females and one male within an age range of 32-63 years old and a weight varying from 60 to $95 \mathrm{~kg}$ (Table 3 ). The study addresses the PK parameters based on a single oral administration of $20 \mathrm{mg}$ OME tablets (racemate) to each volunteer. Blood and saliva samples were collected from 


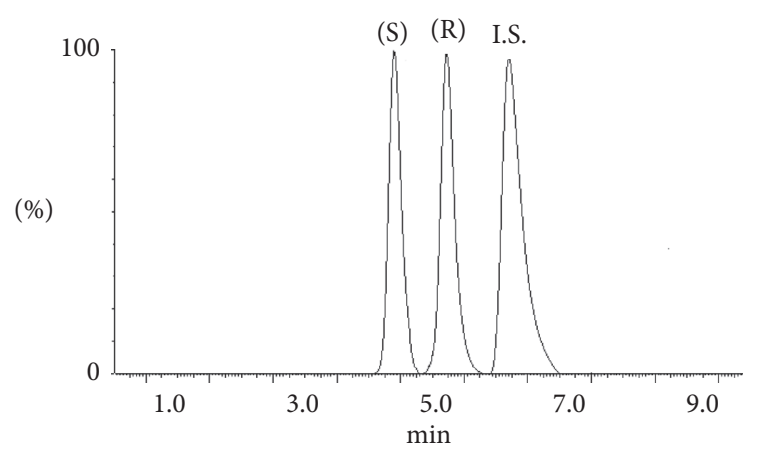

(a)

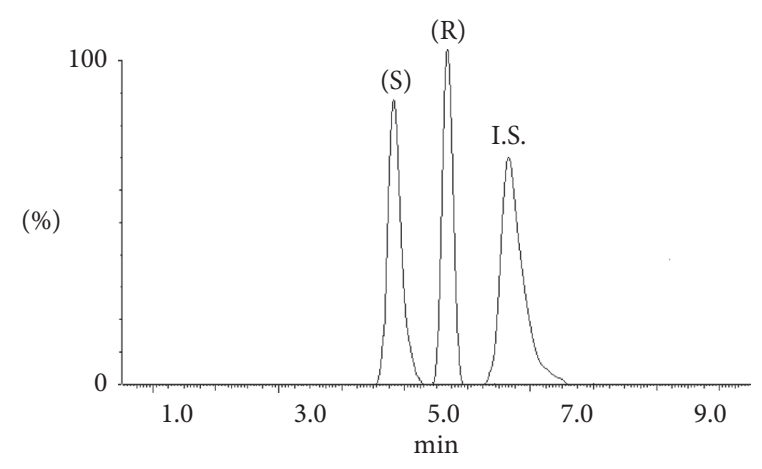

(b)

FIGURE 4: LC-MS/MS chromatograms of OME enantiomers in plasma (a) and oral fluid sample (b).

TABLE 2: Determination of OME with different methods.

\begin{tabular}{|c|c|c|c|c|c|}
\hline Method & $\begin{array}{c}\text { Linear } \\
\text { range } \\
(\mathrm{ng} / \mathrm{mL})\end{array}$ & Matrix (OM form) & Sample preparation method & $\begin{array}{c}\text { Extraction efficiency } \\
(\%)\end{array}$ & Ref. \\
\hline RP-HPLC & $2-2000$ & Human plasma (racemate) & LLE & 84 & {$[10]$} \\
\hline HILIC-MS/MS & $2-1000$ & Human plasma (racemate) & LLE & $87-98$ & {$[11]$} \\
\hline $\begin{array}{l}\text { UHPLC-MS/ } \\
\text { MS }\end{array}$ & $10-500$ & Rat plasma (racemate) & $\begin{array}{l}\text { Protein precipitation and } \\
\text { LLE }\end{array}$ & 87 & {$[36]$} \\
\hline LC-UV & $50-1000$ & Human plasma (enantiomers) & SPE & $93-94$ & {$[37]$} \\
\hline LC-MS/MS & $1.25-2500$ & Human plasma (enantiomers) & Protein precipitation (PP) & $105-106$ & [38] \\
\hline LC-MS/MS & $25-500$ & $\begin{array}{l}\text { Human plasma, oral fluid } \\
\text { (enantiomers) }\end{array}$ & MEPS & $94-98$ & $\begin{array}{l}\text { Present } \\
\text { study }\end{array}$ \\
\hline
\end{tabular}

TABLE 3: The physical data of selected volunteers.

\begin{tabular}{lccc}
\hline Subject no. & Sex & Weight & Age \\
\hline 1 & Male & 80 & 56 \\
2 & Female & 95 & 59 \\
3 & Female & 60 & 63 \\
4 & Female & 70 & 32 \\
& Average & 76.25 & 52.5 \\
\hline
\end{tabular}

each individual throughout $24 \mathrm{~h}$ after administration of the drug.

The OME enantiomers in human plasma and saliva samples were analyzed by the present method. The PK characteristics being expressed by its parameters, such as elimination rate constant $\left(k_{\mathrm{e}}\right)$ and half-time $\left(t_{1 / 2}\right)$, were then estimated using the concentration-time profile data for each enantiomer. $C_{\max }$ and $T_{\max }$ were estimated directly from the concentration-time curves.

The PK parameters were evaluated for each enantiomer in the four healthy persons by calculating the mean values (Table 4). The results showed that the (S)-enantiomer has higher $C_{\max }$ and exposure as expressed as the area under the concentration-time curve (AUC) PK values compared to that found for the (R)-enantiomer ((R)-OME) by approximately 1.5 and 1.3 -fold, respectively. $C_{\max }$ for both enantiomers reached $2.5 \mathrm{~h}$ after drug administration $\left(T_{\max }\right)$ (Figure $\mathrm{S} 1$ ). The elimination rate of the (R)-
TABLE 4: Main pharmacokinetic parameters of OME enantiomers in plasma and oral fluid following single oral dose of $20 \mathrm{mg}$ to healthy volunteers $(n=4)$.

\begin{tabular}{lcccc}
\hline \multirow{2}{*}{ Parameter } & \multicolumn{2}{c}{ Plasma } & \multicolumn{2}{c}{ Oral fluid } \\
& $(\mathrm{R})-\mathrm{OME}$ & $(\mathrm{S})$-OME & $(\mathrm{R})$-OME & $(\mathrm{S})$-OME \\
\hline$C_{\max }\left(\mathrm{ng} \cdot \mathrm{mL}^{-1}\right)$ & 62.44 & 97.24 & 40.01 & 43.61 \\
$T_{\max }(\mathrm{h})$ & 2.50 & 2.50 & 2.00 & 2.00 \\
$k_{\mathrm{el}}\left(\mathrm{h}^{-1}\right)$ & 0.267 & 0.207 & 1.811 & 1.151 \\
$t_{1 / 2}(\mathrm{~h})$ & 2.59 & 3.35 & 0.58 & 0.60 \\
$\mathrm{AUC}_{0-24}$ & 384.86 & 510.80 & 125.99 & 130.66 \\
$\mathrm{AUC}_{0-\infty}$ & 411.72 & 544.25 & 128.59 & 134.79 \\
$\mathrm{CL} / f\left(\mathrm{~L} \cdot \mathrm{kg}^{-1} \cdot \mathrm{h}^{-1}\right)$ & 0.631 & 0.477 & 1.944 & 1.855 \\
$\mathrm{VD} / f\left(\mathrm{~L} \cdot \mathrm{kg}^{-1}\right)$ & 2.361 & 2.306 & 1.073 & 1.611 \\
\hline
\end{tabular}

enantiomer was slightly higher than that of the (S)-enantiomer. The half-time $\left(t_{1 / 2}\right)$ of the $S$-enantiomer was approximately $0.7 \mathrm{~h}$ compared to that obtained for the $\mathrm{R}$-enantiomer. The higher oral clearance rate $(\mathrm{CL} / f)$ of the (R)-enantiomer provides a proof of the stereoselective metabolism. The $(\mathrm{Vd} / f)$ values of both enantiomers were approximately similar indicating high tissue distribution of both forms. The $(\mathrm{S})$-enantiomer showed higher $C_{\max }$ than that of (R)-OME with similar AUC0-24 values (Table 4). Both enantiomers showed faster $T_{\max }$ values in saliva compared to that seen in plasma, while both enantiomers attained higher AUC and $C_{\max }$ values in plasma than that in saliva. 


\section{Conclusion}

A new method based on LC-MS/MS has been developed and validated for the assay of the enantiomers of OME in human plasma and oral fluid samples. Both plasma and oral fluid samples were treated with MEPS technique before analysis. Quantitative analysis of OME enantiomers in both matrices were achieved successfully with good accuracy and precision. The method demonstrated to be an excellent method for the chiral separation of OME enantiomers as well as for the monitoring of OME in plasma. Therefore, the method was used for the PK study of the omeprazole enantiomers.

The evaluation of the PK parameters in oral fluid for omeprazole showed poor correlation to those in plasma. However, the use of oral fluid serves as a fast and easy sampling medium for screening of the drug's therapeutic levels. On the other hand, possible enzymatic actions in the oral fluid and the intersubject variations in the enzymatic composition of oral fluid $s$ can contribute to the variation in the drug levels that requires further investigation.

\section{Data Availability}

The data used to support the findings of this study are available in supplementary information files.

\section{Conflicts of Interest}

The authors declare that they have no conflicts of interest.

\section{Supplementary Materials}

Figure S1The mean plasma concentration-time curve of $(\mathrm{a})(\mathrm{R})$-omeprazole and (b)(S)-omeprazole in females compared to male volunteers after $24 \mathrm{~h}$ of the administration of a single oral tablet containing $20 \mathrm{mg}$ of omeprazole racemate. Table S1Stability data of omeprazole enantiomers in plasma and saliva. (Supplementary Materials)

\section{References}

[1] L. S. Goodman, L. L. Brunton, A. Gilman, B. Chabner, and B. C. Knollmann, Goodman and Gilman's: The Pharmacological Basis of Therapeutics, McGraw-Hill Medical, NY, USA, 12th edition, 2011.

[2] W. Asghar, E. Pittman, and F. Jamali, "Comparative efficacy of esomeprazole and omeprazole: racemate to single enantiomer switch," DARU Journal of Pharmaceutical Sciences, vol. 23, pp. 50-57, 2015.

[3] A. Rahman, M. Rashedul Haque, Z. Sultan, M. M. Rahman, and M. A. Rashid, "Enantiomeric determination of omeprazole and esomeprazole by a developed and validated chiral HPLC method and stability studies by microthermal analysis," Journal of Pharmaceutical Sciences, vol. 16, no. 2, pp. 221-233, 2017.

[4] T. Lind, L. Rydberg, A. Kylebäck et al., "Esomeprazole provides improved acid control vs. omeprazole in patients with symptoms of gastro-oesophageal reflux disease," Alimentary Pharmacology \& Therapeutics, vol. 14, no. 7, pp. 861-867, 2000.
[5] A. Choudhury, S. Das, S. Bahadur, S. Saha, and A. Roy, "Formulation and evaluation of omeprazole tablets for duodenal ulcer," Indian Journal of Pharmaceutical Sciences, vol. 72, no. 4, pp. 491-494, 2010.

[6] A. M. Cairns, R. H.-Y. Chiou, J. D. Rogers, and J. L. Demetriades, "Enantioselective high-performance liquid chromatographic determination of omeprazole in human plasma," Journal of Chromatography B: Biomedical Sciences and Applications, vol. 666, no. 2, pp. 323-328, 1995.

[7] H. Elmogy and M. Abdel-Rehim, "Oral fluid as an alternative specimen to plasma for drug bioanalysis: a review," TrAC Trends in Analytical Chemistry, vol. 83, pp. 70-79, 2016.

[8] H. Kanazawa, A. Okada, M. Higaki, H. Yokota, F. Mashige, and K. Nakahara, "Stereospecific analysis of omeprazole in human plasma as a probe for CYP2C19 phenotype," Journal of Pharmaceutical and Biomedical Analysis, vol. 30, no. 6, pp. 1817-1824, 2003.

[9] M. Ishii, M. Sato, M. Ogawa, T. Takubo, K. i. Hara, and Y. Ishii, "Simultaneous determination of omeprazole and its metabolites (5'-hydroxyomeprazole and omeprazole sulfone) in human plasma by liquid chromatography-tandem mass spectrometry," Journal of Liquid Chromatography \& Related Technologies, vol. 30, no. 12, pp. 1797-1810, 2007.

[10] N. L. Rezk, K. C. Brown, and A. D. M. Kashuba, "A simple and sensitive bioanalytical assay for simultaneous determination of omeprazole and its three major metabolites in human blood plasma using RP-HPLC after a simple liquid-liquid extraction procedure," Journal of Chromatography B, vol. 844, no. 2, pp. 314-321, 2006.

[11] Q. Song and W. Naidong, "Analysis of omeprazole and 5-OH omeprazole in human plasma using hydrophilic interaction chromatography with tandem mass spectrometry (HILICMS/MS)-eliminating evaporation and reconstitution steps in 96-well liquid/liquid extraction," Journal of Chromatography B, vol. 830, no. 1, pp. 135-142, 2006.

[12] A. Abdel-Rehim and M. Abdel-Rehim, "Dried saliva spot as a sampling technique for saliva samples," Biomedical Chromatography, vol. 28, no. 6, pp. 875-877, 2014.

[13] H. Stenhoff, A. Blomqvist, and P.-O. Lagerström, "Determination of the enantiomers of omeprazole in blood plasma by normal-phase liquid chromatography and detection by atmospheric pressure ionization tandem mass spectrometry," Journal of Chromatography B: Biomedical Sciences and Applications, vol. 734, no. 2, pp. 191-201, 1999.

[14] Q. B. Cass, V. V. Lima, R. V. Oliveira, N. M. Cassiano, A. L. G. Degani, and J. Pedrazzoli, "Enantiomeric determination of the plasma levels of omeprazole by direct plasma injection using high-performance liquid chromatography with achiral-chiral column-switching," Journal of Chromatography B, vol. 798, no. 2, pp. 275-281, 2003.

[15] R. Said, M. Kamel, A. El-Beqqali, and M. Abdel-Rehim, "Microextraction by packed sorbent for LC-MS/MS determination of drugs in whole blood samples," Bioanalysis, vol. 2, no. 2, pp. 197-205, 2010.

[16] M. Abdel-Rehim, "Recent advances in microextraction by packed sorbent for bioanalysis," Journal of Chromatography A, vol. 1217, no. 16, pp. 2569-2580, 2010.

[17] M. M. Moein, A. Abdel-Rehim, and M. Abdel-Rehim, "Microextraction by packed sorbent (MEPS)," TrAC Trends in Analytical Chemistry, vol. 67, pp. 34-44, 2015. 
[18] M. Abdel-Rehim, "Microextraction by packed sorbent (MEPS): a tutorial," Analytica Chimica Acta, vol. 701, no. 2, pp. 119-128, 2011.

[19] M. M. Moein, A. Abdel-Rehim, and M. Abdel-Rehim, "Online determination of sarcosine in biological fluids utilizing dummy molecularly imprinted polymers in microextraction by packed sorbent," Journal of Separation Science, vol. 38, no. 5, pp. 788-795, 2015.

[20] Z. Altun, A. Hjelmström, M. Abdel-Rehim, and L. G. Blomberg, "Surface modified polypropylene pipette tips packed with a monolithic plug of adsorbent for highthroughput sample preparation," Journal of Separation Science, vol. 30, no. 12, pp. 1964-1972, 2007.

[21] M. Abdel-Rehim, "New trend in sample preparation: online microextraction in packed syringe for liquid and gas chromatography applications," Journal of Chromatography B, vol. 801, no. 2, pp. 317-321, 2004.

[22] L. A. Tabak, "A revolution in biomedical assessment: the development of salivary diagnostics," Journal of Dental Education, vol. 65, no. 12, pp. 1335-1339, 2002.

[23] S. Chiappin, G. Antonelli, R. Gatti, and E. F. De Palo, "Saliva specimen: a new laboratory tool for diagnostic and basic investigation," Clinica Chimica Acta, vol. 383, no. 1-2, pp. 30-40, 2007.

[24] Y.-H. Lee and D. T. Wong, "Oral fluid: an emerging biofluid for early detection of diseases," American Journal of Dentistry, vol. 22, no. 4, pp. 241-248, 2009.

[25] D. Malamud, "Saliva as a diagnostic fluid," Dental Clinics of North America, vol. 55, no. 1, pp. 159-178, 2011.

[26] N. Spielmann and D. Wong, "Saliva: diagnostics and therapeutic perspectives," Oral Diseases, vol. 17, no. 4, pp. 345-354, 2011.

[27] L. F. Hofman, "Human saliva as a diagnostic specimen," The Journal of Nutrition, vol. 131, no. 5, pp. 1621s-1625 s, 2001.

[28] J. Liu and Y. Duan, "Saliva: a potential media for disease diagnostics and monitoring," Oral Oncology, vol. 48, no. 7, pp. 569-577, 2012.

[29] C. Streckfus and L. Bigler, "Saliva as a diagnostic fluid," Oral Diseases, vol. 8, no. 2, pp. 69-76, 2002.

[30] G. Iorgulescu, "Oral fluid between normal and pathological. Important factors in determining systemic and oral health," Journal of Medicine and Life, vol. 2, no. 3, pp. 303-307, 2009.

[31] Q. B. Cass, A. L. G. Degani, and N. Cassiano, "The use of a polysaccharide-based column on multimodal elution," Journal of Liquid Chromatography \& Related Technologies, vol. 23, no. 7, pp. 1029-1038, 2000.

[32] T. Andersson and L. Weidolf, "Stereoselective disposition of proton?? pump inhibitors," Clinical Drug Investigation, vol. 28 , no. 5, pp. 263-279, 2008.

[33] T. Andersson, K. RÖhss, E. Bredberg, and M. Hassan-Alin, "Pharmacokinetics and pharmacodynamics of esomeprazole, the S-isomer of omeprazole," Alimentary Pharmacology \& Therapeutics, vol. 15, no. 10, pp. 1563-1569, 2001.

[34] H. Inoue, K. Ono, W. Masuda et al., "Gender difference in unstimulated whole saliva flow rate and salivary gland sizes," Archives of Oral Biology, vol. 51, no. 12, pp. 1055-1060, 2006.

[35] Food and Drug Administration, "FDA policy statement for the development of new stereoisomeric drugs," Chirality, vol. 4, no. 5, pp. 338-340, 1992.

[36] Z. Wang, W. Sun, Z.-F. Lin et al., "A UHPLC-MS/MS method coupled with liquid-liquid extraction for the quantitation of phenacetin, omeprazole, metoprolol, midazolam and their metabolites in rat plasma and its application to the study of four CYP450 activities," Journal of Pharmaceutical and Biomedical Analysis, vol. 163, pp. 204-210, 2019.

[37] R. M. Orlando and P. S. Bonato, "Simple and efficient method for enantioselective determination of omeprazole in human plasma," Journal of Chromatography B, vol. 795, no. 2, pp. 227-235, 2003.

[38] L. N. Sun, Y. Zhang, Y. Q. Yang et al., "Simultaneous enantioselective determination of omeprazole, rabeprazole, lansoprazole, and pantoprazole enantiomers in human plasma by chiral liquid chromatography-tandem mass spectrometry," Journal of Separation Science, vol. 43, no. 16, pp. 3183-3196, 2020. 